\title{
Vocal cord dysfunction: what do we know?
}

\author{
K. Kenn* and R. Balkissoon\#
}

ABSTRACT: Vocal cord dysfunction (VCD) is a disorder caused by episodic unintentional paradoxical adduction of the vocal cords, which may induce acute severe dyspnoea attacks not responsive to conventional asthma therapy. The aetiology of VCD is complex and often multifactorial. The essential pathophysiology is that of a hyperfunctional laryngeal reflex to protect the lower airway as a result of any combination of post-nasal drip, gastro-oesophageal reflux, laryngopharyngeal reflux and/or psychological conditions. Laryngoscopic demonstration of the paradoxical motion while wheezing or stridorous is considered the diagnostic gold standard. Speech therapy, including the use of special relaxed-throat breathing patterns is effective for VCD that is purely of the functional nature. Knowledge of the clinical features of VCD and identifying factors that may be contributing to the development of VCD can provide adequate clues to the correct diagnosis and management.

KEYWORDS: Dyspnoea attacks, intractable asthma, vocal cord dysfunction

$\mathbf{R}$ efractory or severe asthma is estimated to afflict only $5-10 \%$ of all asthma patients but consumes a disproportionate amount of healthcare costs [1]. Treatment failure often leads to an escalation of drug therapy, including oral corticosteroids, which often produce critical side-effects, including fluid retention, acne, adrenal insufficiency, weight gain, loss of bone density, cataracts, stria and other cushingoid features that can be quite distressing. When evaluating these patients, there is a broad differential diagnosis that can be considered including noncompliance/ nonadherence, ongoing allergen exposure, chronic rhinosinusitis and/or gastro-oesophageal reflux (GER) disease. Paradoxical vocal fold motion disorder, more popularly known as vocal cord dysfunction (VCD), can mimic asthma and is gaining increasing recognition as a common cause of respiratory symptoms in children and in adults often diagnosed with steroid refractory asthma. This article will review our current understanding of the epidemiology, pathogenesis, diagnosis and management of VCD.

\section{DEFINITIONS}

While there is no consensus with regard to the definition of VCD, we here define it as an intermittent extrathoracic airway obstruction mainly during inspiration leading to dyspnoea of varying intensity [2]. In Europe, otolaryngologists sometimes use the term VCD to describe various voice disorders, such as spastic dysphonia. This article will use the term VCD to refer to the patients typically seen by pulmonologists and allergists with respiratory symptoms.

\section{HISTORICAL PERSPECTIVE}

The first modern case report was published by PATTERSON et al. [3] in 1974 who demonstrated laryngoscopic evidence of VCD, which he termed "Munchausen's stridor". In 1983 CHRIsTOPHER et al. [4] published a seminal paper that described a group of patients evaluated for severe asthma who were found to have VCD. They described a group largely comprised of females between the ages of 20-40 yrs with histories of physical and/ or sexual abuse. This study reported that a high proportion of these females were healthcare workers. NEWMAN et al. [5] conducted a retrospective chart review of 95 patients with a diagnosis of intractable asthma and found that $10 \%$ of the patients had VCD alone and $30 \%$ of the patients had VCD with asthma. Since these early studies, there has been increased awareness and interest in understanding the risk factors, pathogenesis, diagnosis and optimum management of this condition.

\section{EPIDEMIOLOGY OF VCD}

The lack of uniformity in definitions and diagnostic criteria for VCD make studies of the incidence, prevalence and risk factors problematic. There have been no prospective cohort studies to assess the development of new cases, hence we have no information regarding the true incidence of VCD per se. There are however
AFFILIATIONS

*Dept of Pulmonology, Allergology, Hospital Klinikum Berchtesgadener Land, Schoenau am Koenigssee, Germany.

${ }^{*}$ National Jewish Medical and Research Center, Denver, CO, USA.

CORRESPONDENCE

K. Kenn

Klinikum Berchtesgadener Land Dept of Pulmonology Malterhoeh 1

D 83471 Schoenau am Koenigssee E-mail: KKenn@schoen-kliniken.de

Received:

Dec 062009

Accepted after revision:

Jan 242010 
several studies, both prospective and retrospective, that have provided estimates of the prevalence of VCD in various clinical settings and patient populations.

CicCOlella et al. [6] reported a prevalence of $2.5 \%$ and JAIN et al. [7] a prevalence of $22 \%$ in patients with frequent emergency room visits due to dyspnoea of sudden onset. In a prospective German study of 1,028 patients admitted to a rehabilitation unit due to breathing problems in the prior year, a prevalence of $2.8 \%$ was observed [8]. BRUGMAN [9] conducted a systematic literature review and found that of 1,530 patients reported to have VCD, there was a broad age range, from infant to $82 \mathrm{yrs}$, and the female to male ratio was 3:1. MorRIS et al. [10] also conducted a systematic literature review that encompassed 1,161 patients diagnosed with VCD and found a female to male ratio of 2:1. Hence, while the literature does indeed support VCD as being more common in females, more recent prospective studies demonstrate that by no means is it uncommon in males.

VCD has certainly been reported in children as well. BRUGMAN [9] reported in her review of 1,530 VCD cases that 35\% were children with a median age of 14 yrs. She reported that $75 \%$ of the children with refractory asthma had associated VCD symptoms and a VCD prevalence of up to $14 \%$ in children and adolescents being hospitalised with a diagnosis of asthma.

There is a subset of patients with VCD that reports exertion as their major trigger for dyspnoea and are typically diagnosed with exercise-induced asthma, but don't respond to typical therapy. In a prospective study with active duty US soldiers suffering from exertional dyspnoea, VCD was found as an underlying disorder in up to $15 \%$ [11]. About 5\% of elite athletes at the US Olympic Training Centre in Lake Placid showed inspiratory stridor during exercise testing in cold, dry, ambient conditions [12], but lung function tests or laryngoscopy were not performed to confirm the diagnosis of VCD in this study.

PERKNER et al. [13] identified a group of individuals originally described as having reactive airways dysfunction syndrome from irritant occupational exposures, who subsequently were found to have negative methacholine challenges but laryngoscopic evidence of VCD. These patients were diagnosed as having irritant-associated VCD.

Hence, it is now evident that there are a number of groups that appear to be at increased risk of developing VCD beyond the stereotypical abused female or female health care worker. As outlined, additional groups identified, who may be at increased risk, include elite athletes, military recruits and individuals who have had high level irritant exposures [11].

\section{PATHOPHYSIOLOGY: CURRENT CONCEPTS}

A review of the current theories with regard to the pathogenesis of VCD allows us to put into context the observed association between GER, laryngopharyngeal reflux (LPR), post-nasal drip, asthma and psychological factors in patients with VCD. The larynx is the gateway to the trachea with two sets of folds (also referred to as cords); the false vocal folds and the true vocal folds form the slit-like opening to the trachea called the glottis. During inspiration, the cross-sectional area between the vocal folds widens especially during deep inspiration [14] and it narrows slightly $(<30 \%)$ during expiration. Sensory receptors from the nose to the bronchi detect irritant stimuli that trigger cough and glottic closure reflexes that are central to protecting the lungs from exogenous noxious agents. The rise in subglottic pressure against a closed glottis is important for the cough reflex but also provides a form of automatic positive end-expiratory pressure (PEEP) by increasing intra-alveolar pressure. This form of auto-PEEP has been used by severe asthmatics and those with emphysema to prevent or reduce airway collapse during expiration and thus promote better emptying. Hence, mid-to-late expiratory closure as a means of creating auto-PEEP should not be regarded as dysfunctional or maladaptive.

Given the role of the larynx to protect the trachea and lungs from noxious inhalants it is understandable that chronic postnasal drip may lead to increased laryngeal sensitivity and consequent laryngeal hyperresponsiveness [14, 15]. BuccA et al. [15] followed a group of patients with chronic rhinosinusitis and verified their upper airway hyperresponsiveness by measuring the concentration of histamine that caused a $25 \%$ drop in the mid-inspiratory flow (MIF50). They found that 76 (72\%) out of 106 patients had extrathoracic hyperresponsiveness and $46(52 \%)$ patients had both bronchial hyperresponsiveness and extrathoracic hyperresponsiveness. Interestingly, they found a reduction in the extrathoracic hyperresponsiveness and bronchial hyperresponsiveness after treatment with antibiotics and inhaled nasal steroids for 2 weeks. Another mechanism by which non-allergic rhinitis may trigger VCD is by alterations in olfaction and/or trigeminal chemoreception, particularly increased chemosensory sensitivity as is sometimes seen after irritant induced rhinitis. Olfactory triggers may cause augmentation of the glottic closure reflex and trigger VCD attacks at lower odour thresholds than might otherwise occur [16].

GER refers to the retrograde flow of gastric contents into the oesophagus. GER disease is extremely common and the estimated prevalence is $10-60 \%$ in the general population based on a meta-analysis of studies using $\mathrm{pH}$ probes [17]. It is reported to be as high as $60 \%$ in the asthma population [18-23]. While there is evidence that the presence of acid in the oesophagus can cause bronchoconstriction and cough, the primary connection between GER and VCD may be LPR.

LPR refers to the regurgitant flow of gastric contents including acid and digestive enzymes such as pepsin into the laryngopharynx leading to symptoms of hoarseness, cough, halitosis, dysphagia, throat clearing and VCD [23]. The epidemiology of LPR is not well established, partially because of relatively few studies examining the question, but more importantly because there are no clear gold standard diagnostic criteria. One study showed that $86 \%$ of 105 healthy adults had some signs of reflux on laryngoscopic examination [24]. The refluxate may cause direct damage to the laryngeal mucosa leading to symptoms. It is important to note that the laryngeal mucosa may be damaged by the refluxate either because it is not protected by the peristaltic motion or because it is not buffered by salivary bicarbonate. The lower laryngeal mucosa is different from that of the oesophagus and the stomach, such that much less acid (and/or pepsin) regurgitation is required to cause significant damage and symptoms. While it is reported that $\mathrm{pH}$ 
drops below 4 are associated with oesophageal damage, it is recognised that the deleterious actions of pepsin can occur with $\mathrm{pH}$ levels as high as 6 [17]. Previous studies have suggested that 50 episodes of reflux are at the upper limits of normal, however three episodes of laryngeal reflux over a week are capable of leading to significant damage in the laryngeal area [23, 25, 26, 27].

There are studies emerging that suggest that the larynx can become hypersensitive [28] and may demonstrate evidence of a sensory neuropathy [29] or increases in nerve growth factor [30]. SHUSTERMAN and co-workers [31-33] have demonstrated the increased odour intolerance of individuals with upper airway dysfunction.

While it is clear that there is a subset of patients with VCD that have primarily a psychological or so called functional disorder, there are now numerous reports linking GER disease, LPR and post-nasal drip with VCD [28, 34, 35]. While the precise mechanisms have not been definitively established it may be that the damage to the laryngeal mucosa leads to an accentuation of the glottic closure reflex but this requires further study. This mechanism is also felt to be important in patients who develop VCD after irritant exposures [31, 36-39].

In summary, while the above discussed pathophysiology may be seen as state of the art as far as our current understanding. It must be said, that these are still unproven concepts and we need prospective studies to clarify the underlying mechanisms for VCD.

\section{CLINICAL EVALUATION OF VCD \\ History}

Obtaining the history for possible VCD is usually in the context of assessing a patient for refractory asthma or chronic cough. The history of a patient with VCD may sound very similar to that of an asthmatic with reports of shortness of breath, wheezing and cough with exposure to exercise or a number of irritants. VCD related dyspnoea is characterised by sudden onset, short duration (mostly $<2 \mathrm{~min}$ ) and is typically selflimited. Classically inspiration is affected and the narrowing is localised in the throat or the upper part of the trachea [8]. VCD patients may report less chest tightness than asthmatics but that is nonspecific (table 1). When patients localise symptoms to the throat it argues against asthma as the explanation of symptoms.

Most VCD patients will have been given a diagnosis of asthma and have received frequent prednisone bursts with only marginal or no response. NEWMAN et al. [5] reported that a group of retrospectively evaluated patients with VCD only had an average daily dose of $29.2 \mathrm{mg}$ of prednisolone, medical utilisation was high with 9.7 emergency visits and 5.9 admissions in the year prior to evaluation and $28 \%$ of these patients had been previously intubated. Some patients will report audible wheezing during inspiration and/or expiration. Questions should be directed to detecting the possible evidence of post-nasal drip and GER and LPR. The reported presence of allergies, nasal congestion, throat clearing, hoarseness, chronic cough, headaches, heartburn, indigestion, water brash, throat burning, halitosis, increased cough lying flat and cough worse at night are all clues to post-nasal drip and/or GER disease as likely contributing factors.

Psychological assessment should include a query regarding any history of psychological distress or any history of abuse. This is extremely important for the patients where a primary functional disorder is suspected. Furthermore, a number of patients with other problems, such as post-nasal drip and/or LPR will have psychological issues, such as anxiety and/or depression as a result of being told they have a condition (asthma) that is potentially life threatening for which they seem to be getting worse despite aggressive therapy.

\section{Physical exam}

Physical exam may be relatively unremarkable except for detection of laryngeal wheezing and/or stridor at the time of an acute attack. One should also look for stigmata of posterior

\section{TABLE 1 Diagnostic approach in vocal cord dysfunction}

Evaluation/test

History (nonspecific)

\section{Lung function tests}

Symptom-free period

Acute dyspnoea (may be provoked by methacholine/ histamine challenge, exercise, irritant challenge)

ENT examination (laryngoscopy)

Evaluation of reflux GER disease/LPR
Typical findings

Throat tightness/closure, globus sensation, chest tightness, audible wheezing (inspiratory versus expiratory), stridor triggers: irritants, exercise trigger poor response or aggravation by inhaled medications (MDI>nebulised)

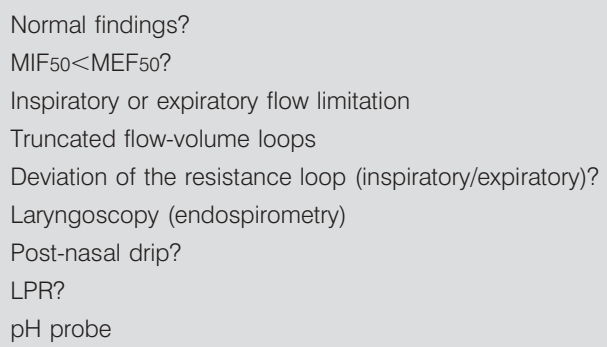


a)

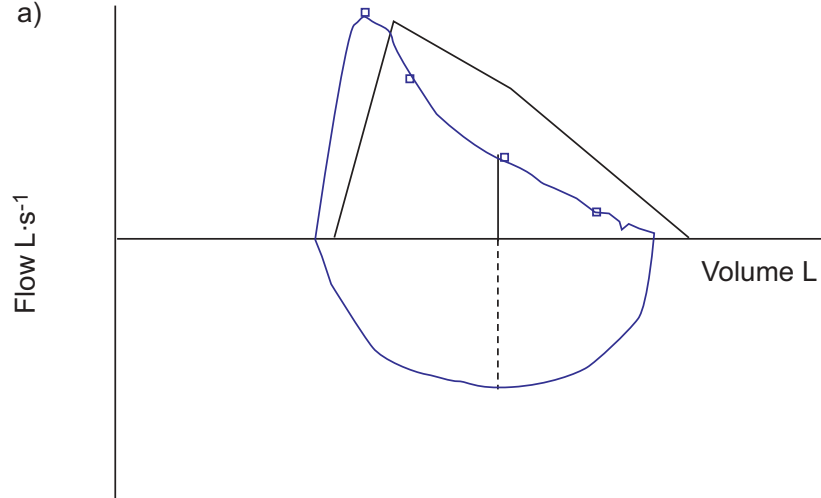

b)

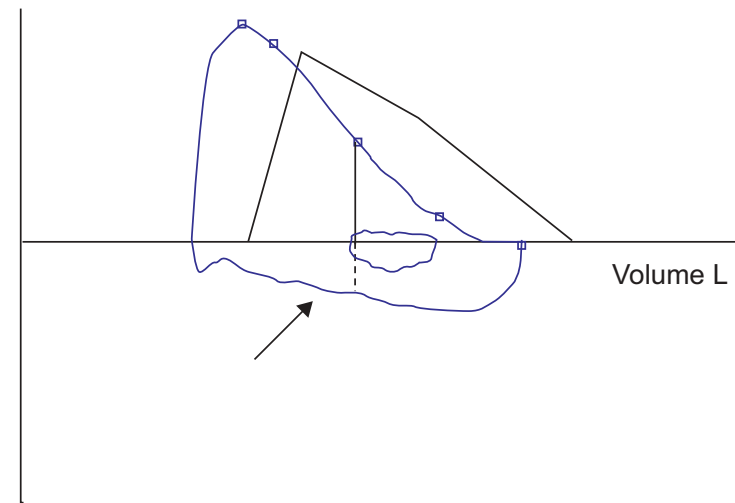

FIGURE 1. a) Pre- and b) post-methacholine changes in flow-volume loop. Inspiratory curve flattened (black arrow) following methacholine challenge leads to reversal in maximal inspiratory flow at $50 \%$ of forced vital capacity (MIF50)/maximal expiratory flow at $50 \%$ of forced vital capacity (MEF50) ratio from a) $>1$ to b) $<1$ (i.e. MIF50 smaller MEF50). —_: MEF50; - - - -: MIF50. Purple line: patient result; black line: predicted result.

nasal drainage or GER (epigastric tenderness and halatosis). The remainder of the physical exam is typically normal for most patients with VCD.

\section{Ancillary tests}

Inbetween attacks spirometry is often completely normal. During a period when the patient is symptomatic spirometry can demonstrate a pattern of variable extrathoracic airway obstruction on the flow-volume loop (truncated inspiratory loop) which is compelling evidence that an obstructive upper airway process may be present. Methacholine challenge can often be used to provoke the paradoxical closure of the upper airway. A maximal inspiratory flow at 50\% of forced vital capacity (MIF50)/ maximal expiratory flow at 50\% of forced vital capacity (MEF50) ratio of $<1$ suggests a VCD related problem (fig. 1). However this parameter has not been worked out for identifying VCD at rest or even during symptoms. Provoking airways with methacholine can induce alterations in the inspiratory portion of the (open) shutter loop during body plethysmography of patients with laryngoscopic confirmed VCD (fig. 2) [14]. The utility of this noninvasive measurement requires validation. Radiographic studies are generally unhelpful in establishing the diagnosis of VCD.

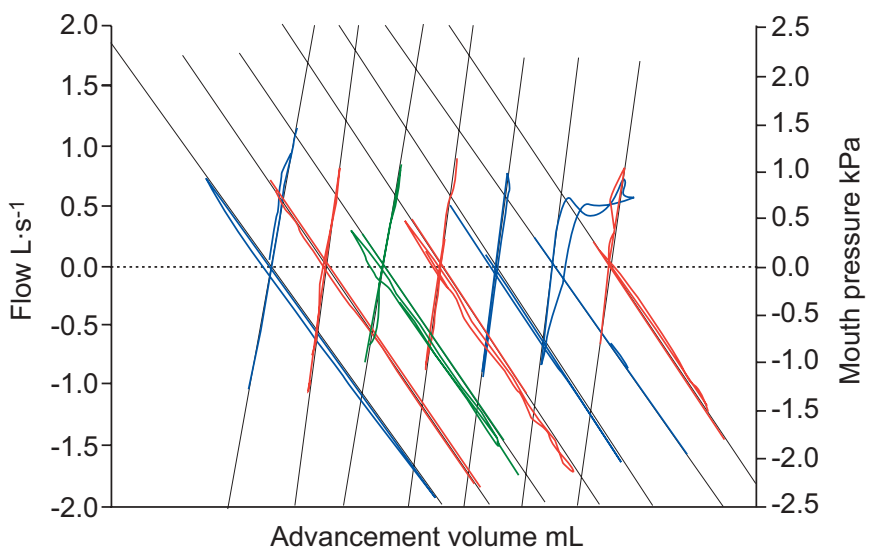

FIGURE 2. Resistance loops (body plethysmography) during methacholine challenge showing abnormal deviation to the right during inspiration at test number 6 , which is spontaneously reversible at test number 7 .
Direct visualisation of the upper airway is the gold standard for making a definitive diagnosis of VCD (fig. 3). Flexible fibreoptic rhinolaryngoscopy may reveal evidence of chronic rhinitis and post-nasal drip and chronic inflammation, with cobble-stoning of the nasal turbinates and or the posterior oropharynx (fig. 4). Asking the patient to do a variety of manoeuvres helps to define their abnormalities. Typically after general visualisation of the pharynx patients are asked to say "EEE", this will normally lead to symmetrical adduction of the vocal folds and help to identify patients who may have paralysis of one of the folds by evidence of asymmetric movements. Asking patients then to quietly breath in and out will sometimes bring out the classic paradoxical adduction of the vocal folds, but often in order to identify VCD, patients must be asked to do a forced vital capacity manoeuvre. It is important to note that despite all these efforts there are some VCD patients in which it is very difficult to get reproducible results in the laboratory setting.

One often can elicit the response by doing provocation studies either with methacholine, with exercise ( \pm cold air) or using irritants, such as perfume or cleaning agents containing chlorine or ammonia [14]. Such nonspecific irritant challenges are often based on the history obtained from patients when asked what are the typical triggers for their symptoms. VCD should not be considered as ruled out until the patient's
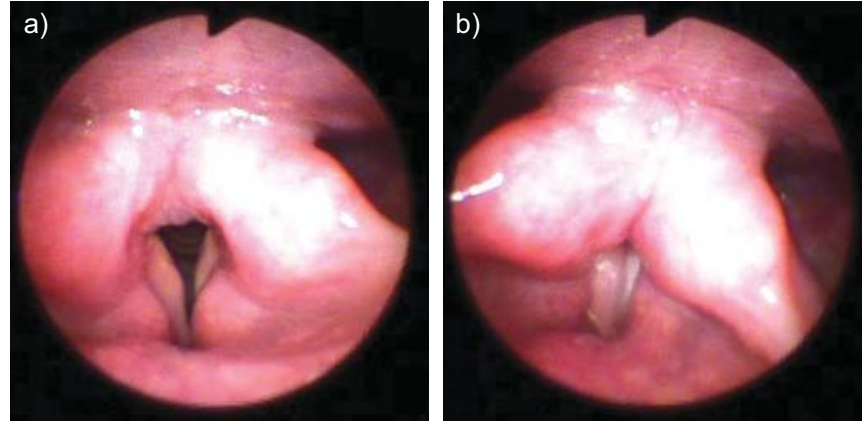

FIGURE 3. Classic vocal cord dysfunction with a) early paradoxical adduction of the vocal folds with formation of a "posterior chink" by b) complete closure of the vocal folds. 


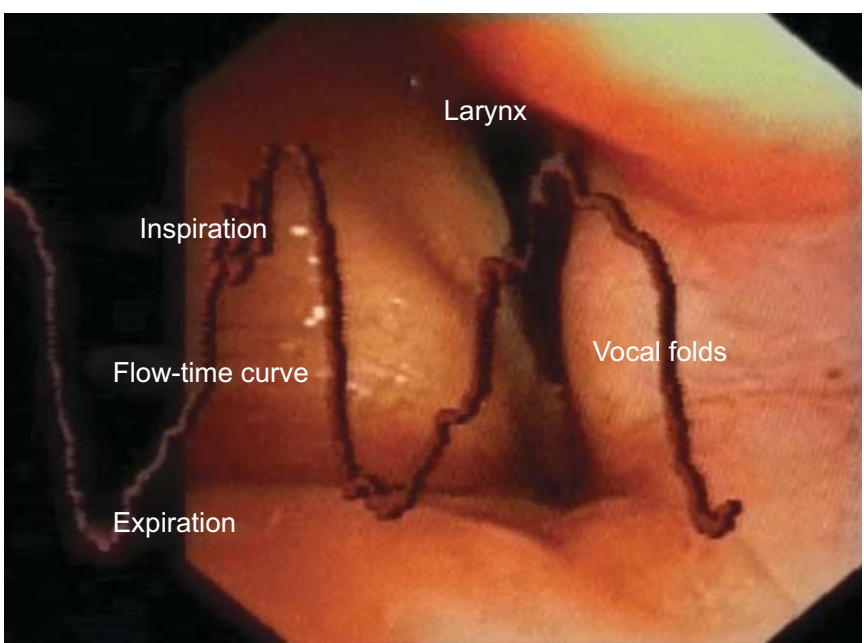

FIGURE 4. Endospirometry presents simultaneous display of flow-time curve (sinusoidal brown line) and laryngoscopic view of the larynx [40]. Airflow limitation and adduction of the vocal cords can be correlated at the same time.

symptoms have been recreated and the vocal cords have been directly visualised during a time patients are having their typical symptoms. Several investigators are looking at newer diagnostic tools to document VCD, including endospirometry, a technique that combines endoscopy timed with spirometric readings (fig. 5) [40].

A review of the diagnostic methods used to diagnose GER and LPR are beyond the scope of this article; however, most patients with LPR are diagnosed on the basis of symptoms and a compatible laryngoscopic exam. The aryepiglottic folds and false cords will often demonstrate chronic inflammation and thickening (fig. 5a), the posterior pharynx will demonstrate cobblestoning (fig. 5b) and the vocal folds may have nodules on them (fig. 5c). Unfortunately these features of reflux laryngitis, posterior laryngeal oedema, true vocal cord oedema, and pseudoulcus, are highly nonspecific [25]. Despite the fact that laryngoscopy is the best evaluation tool currently available there is still no true gold standard for the diagnosis of LPR.
GER disease can be diagnosed on the basis of a compatible history and response to proton pump inhibitor therapy or may require a $\mathrm{pH}$ probe with impedance monitoring to detect high and/or nonacid reflux.

\section{DIFFERENTIAL DIAGNOSIS}

Disorders of laryngeal function may arise as a result of anatomical or neurological abnormalities due to a number of causes. Chronic or slowly progressive airflow obstruction at the level of the larynx may be due to vocal fold polyps or granulomas and tumours, particularly invasive malignancies such as squamous cell carcinomas, lymphomas or thyroid carcinomas. Other aetiologies include papilloma formation leading to internal narrowing in the larynx and trachea and subglottic stenosis or benign thyroid tumours causing extrinsic compression. A significant number of chronic diseases can have laryngeal manifestations including rheumatoid arthritis, lupus, progressive systemic sclerosis, Wegener's granulomatosis and relapsing polychondritis.

The role of uni- or bilateral vocal cord paralysis, e.g. after thyroidectomy may mimic the manifestation of VCD. Unilateral vocal cord paralysis [41] is diagnosed more frequently than bilateral vocal cord paralysis. Patients with bilateral disorder, for unknown reasons, are especially prone to aspiration and severe laryngospasm during sleep. In a significant percentage of patients, a search for the cause of this condition fails to uncover a specific aetiology. The possibility of a cervical or mediastinal malignancy or of an aortic aneurysm encroaching on one recurrent laryngeal nerve (usually the left) must be considered [42].

In contrast to the paroxysmal and variable degree of closure noted with VCD, laryngospasm refers to sustained intense, virtually complete closure of the vocal folds following direct laryngeal stimulation by irritants [43]. The triggers for laryngospasm are not dissimilar from those for VCD and it may very well be that laryngospasm represents the most severe pathophysiological consequences of the glottic closure reflex. Intubation, certain anaesthetics, GER and various other aetiologies have been implicated in inducing laryngospasm [17, 29, 44].
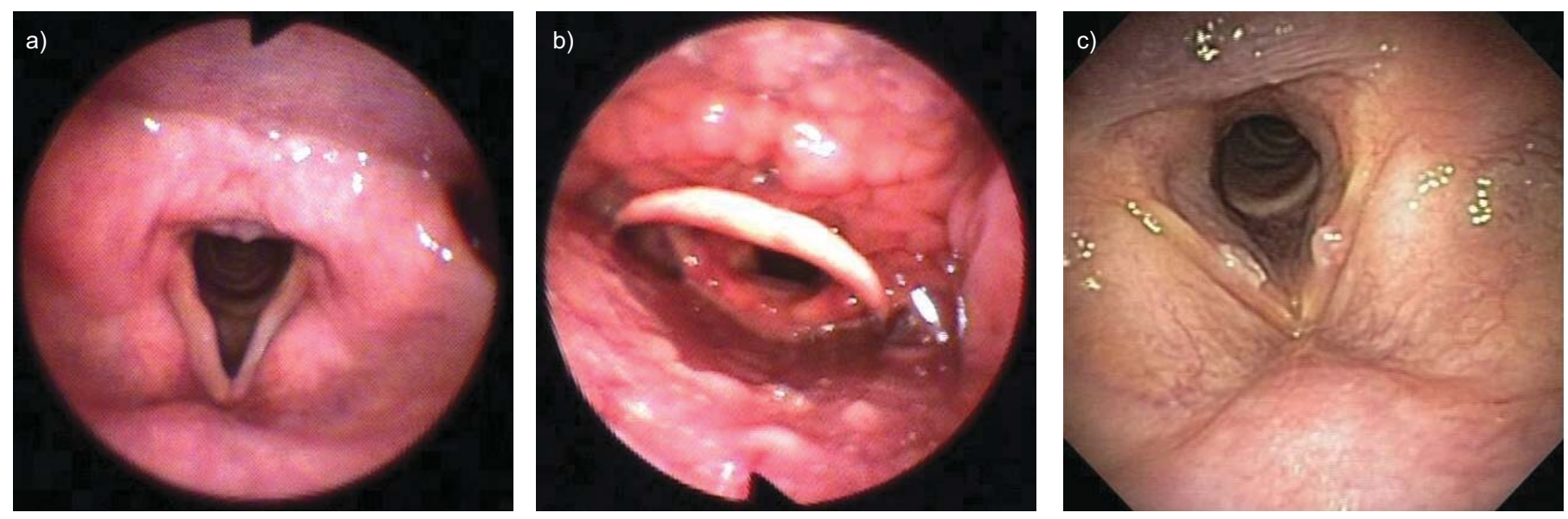

FIGURE 5. Characteristic features of laryngopharyngeal reflux: a) aryepiglottic fold swelling and posterior commissure thickening (pachyderma); b) posterior phayngeal wall cobblestoning; and c) vocal fold nodules. 
TABLE 2 Differences and similarities of asthma and vocal cord dysfunction (VCD)

\begin{tabular}{lcc} 
& Asthma & VCD \\
\hline $\begin{array}{l}\text { Time of onset } \\
\text { Duration }\end{array}$ & Quick (within minutes) & Sudden onset (within seconds) \\
Dyspnoea during & Minutes to hours & Seconds to a few minutes \\
Area of limitation & Expiration & Inspiration \\
Inhaled drug therapy & Thorax, lower airways & Throat \\
Induced by & Highly effective & Often ineffective, aggravating \\
Irritants, stress, exercise
\end{tabular}

Finally, there are functional disorders that must be distinguished from VCD, including spasmodic or muscle tension dysphonia, panic attacks, Munchausen's syndrome [45] and multiple chemical sensitivity (idiopathic environmental intolerance) [46-48] and somatoform disorders [49].

\section{MANAGEMENT OF VCD}

Optimum management of VCD requires the identification of contributing factors (underlying medical problems and psychological factors) and treating these optimally. In addition teaching patients various laryngeal exercises is a task typically conducted by speech and language therapists in the USA and/ or respiratory therapists in Europe. Understanding the complex nature of the pathophysiology of VCD and laryngeal dysfunction underscores the need for a multidisciplinary approach [14]. Physicians (which may include pulmonologists, general internists, otolaryngologists, allergists, occupational medicine specialists and/or psychiatrists), speech therapists, psychosocial medicine, rehabilitation medicine and vocational counsellors may all play useful roles in treating these patients.

Speech and language pathologists/therapists or respiratory therapists provide instruction in techniques of throat relaxation, cough suppression and throat clearing suppression and play a central role in the management and follow-up of VCD. Speech and language therapists or respiratory therapists can coach the patient during controlled irritant challenges with a provocative agent to bring out VCD symptoms, on how to control their laryngeal response or how to abort an acute attack.

Input from psychologists and/or psychiatrists regarding evidence of conversion, panic, anxiety, affective, personality or post-traumatic stress disorders and patient education and reassurance help to reduce anxiety and are extremely important in managing these patients [28, 50]. In addition, follow-up with supportive counselling and the teaching of relaxation and/or biofeedback techniques may also be beneficial. Clinicians should discontinue unnecessary medications, such as bronchodilators and steroids, if coexistent asthma has been ruled out. Treatment for associated GER disease and/or rhinosinusitis may reduce symptoms.

Acute severe episodes of VCD can generally be managed with sedation, and/or Heliox ( $80 \%$ helium $/ 20 \%$ oxygen) [51]. A recent study suggested that a simple continuous positive airway pressure type device providing intermittent positive pressure can resolve an acute attack [52]. Topical lidocaine applied to the larynx may be useful during acute episodes in select patients. In severe cases, superior laryngeal blocks with
Clostridium botulinum toxin have been attempted with variable success [53]. Botulinum toxin injections have been more successful for muscle tension dysphonia than for VCD. Tracheotomy has been used for some patients with severe VCD refractory to conventional therapy, but it is rarely (if ever) indicated.

\section{SUMMARY}

There has been a growing awareness that VCD may mimic or coexist with asthma and lead to over treatment with corticosteroids and the development of significant side-effects. Early and correct diagnosis will avert significant iatrogenic complications (table 2). For many individuals, post-nasal drip and/or LPR are likely the major factors leading to VCD and should be assessed for their presence and treated aggressively. Management of VCD requires identification and treatment of underlying disorders and referral to appropriately trained respiratory therapists who can teach techniques of throat relaxation, cough suppression and throat clearing suppression. There is still a great need for more research into elucidating the underlying pathogenesis and optimum management of VCD.

\section{STATEMENT OF INTEREST}

None declared.

\section{REFERENCES}

1 Wenzel S. Severe asthma in adults. Am J Respir Crit Care Med 2005; 172: $149-160$.

2 Newman KB, Dubester SN. Vocal cord dysfunction: masquerader of asthma. Semin Respir Crit Care Med 1994; 15: 161-167.

3 Patterson R, Schatz M, Horton M. Munchausen's stridor: nonorganic laryngeal obstruction. Clin Allergy 1974; 4: 307-310.

4 Christopher KL, Wood RP, Eckert RC, et al. Vocal cord dysfunction presenting as asthma. N Engl J Med 1983; 308: 1566-1570.

5 Newman KB, Mason UG, Schmaling KB. Clinical features of vocal cord dysfunction. Am J Respir Crit Care Med 1995; 152: 1382-1386.

6 Ciccolella DE, Brennan KJ, Borbely B. Identification of vocal cord dysfunction (VCD) and other diagnoses in patients admitted to an inner city university hospital asthma centre. Am J Respir Crit Care Med 1997; 155: A82.

7 Jain S, Bandi V, Zimmerman J. Incidence of vocal cord dysfunction in patients presenting to emergency room with acute asthma exacerbation. Chest 1997; 11: 243.

8 Kenn K, Schmitz M. Prevalence of vocal cord dysfunction in patients with dyspnea. First prospective clinical study. Am J Respir Crit Care Med 1997; 155: A965.

9 Brugman S. The many faces of vocal cord dysfunction. What 36 years of literature tells us. Am J Respir Crit Care Med 2003; 167: A588. 
10 Morris MJ, Allan PF, Perkins PJ. Vocal cord dysfunction: aetiologies and treatment. Clin Pulm Med 2006; 13: 73-86.

11 Morris MJ, Grabach VX, Deal LE, et al. Evaluation of exertional dyspnea in the active duty patient: the diagnostic approach and the utility of clinical testing. Mil Med 2002; 167: 281-288.

12 Rundell KW, Spiering BA. Inspiratory stridor in elite athletes. Chest 2003; 123: 468-474.

13 Perkner JJ, Fennelly KP, Balkissoon R, et al. Irritant-associated vocal cord dysfunction. J Occup Environ Med 1998; 40: 136-143.

14 Kenn K, Hess M. Vocal cord dysfunction - eine rein pneumologische Erkrankung? [Vocal cord dysfunction - a purely pneumological disease?]. HNO 2004; 52: 103-109.

15 Bucca C, Rolla G, Scappaticci E, et al. Extrathoracic and intrathoracic airway responsiveness in sinusitis. J Allergy Clin Immunol 1995; 95: 52-59.

16 Shusterman D. Critical review: the health significance of environmental odor pollution. Arch Environ Health 1992; 47: 76-87.

17 Merati AL, Lim HJ, Lulalp SO, et al. Meta-analysis of upper probe measurements in normal subjects and patients with laryngopharyngeal reflux. Ann Otol Rhinol Laryngol 2005; 114: 177-182.

18 Field SK, Underwood M, Brant R, et al. Prevalence of gastroesophageal reflux symptoms in asthma. Chest 1996; 109: 316-322.

19 Vincent D, Cohen-Jonathan AM, Leport J, et al. Gastro-oesophageal reflux prevalence and relationship with bronchial reactivity in asthma. Eur Respir J 1997; 10: 2255-2259.

20 Sontag SJ, O'Connell S, Khandelwal S, et al. Most asthmatics have gastroesophageal reflux with or without bronchodilator therapy. Gastroenterology 1990; 99: 613-620.

21 Harding SM, Guzzo MR, Richter JE. The prevalence of gastroesophageal reflux in asthma patients without reflux symptoms. Am J Respir Crit Care Med 2000; 162: 34-39.

22 Harding SM. Gastroesophageal reflux: a potential asthma trigger. Immunol Allergy Clin North Am 2005; 25: 131-148.

23 Harding SM. Recent clinical investigations examining the association of asthma and gastroesophageal reflux. Am J Med 2003; 115: Suppl. 3A, 39S-44S.

24 Koufman JA, Aviv JE, Casiano RR, et al. Laryngopharyngeal reflux: position statement of the committee on speech, voice, and swallowing disorders of the American Academy of Otolaryngology-Head and Neck Surgery. Otolaryngol Head Neck Surg 2002; 127: 32-35.

25 Hicks DM, Ours TM, Abelson TI, et al. The prevalence of hypopharynx findings associated with gastroesophageal reflux in normal volunteers. J Voice 2002; 16: 564-579.

26 Johnston N, Bulmer D, Gill GA, et al. Cell biology of laryngeal epithelial defenses in health and disease: further studies. Ann Otol Rhinol Laryngol 2003; 112: 481-491.

27 Axford SE, Sharp N, Ross PE, et al. Cell biology of laryngeal epithelial defenses in health and disease: preliminary studies. Ann Otol Rhinol Laryngol 2001; 110: 1099-1108.

28 Morrison M, Rammage L, Emami A. The irritable larynx syndrome. J Voice 1999; 13: 447-455.

29 Lee B, Woo P. Chronic cough as a sign of laryngeal sensory neuropathy: diagnosis and treatment. Ann Otol Rhinol Laryngol 2005; 114: 253-257.

30 Millqvist E, Ternesten-Hasséus E, Ståhl A, et al. Changes in levels of nerve growth factor in nasal secretions after capsaicin inhalation in patients with airway symptoms from scents and chemicals. Environ Health Perspect 2005; 113: 849-852.
31 Balkissoon R, Shusterman D. Occupational upper airway disorders. Semin Respir Crit Care Med 1999; 20: 569-580.

32 Shusterman D, Balmes J. Measurement of nasal irritant sensitivity to pulsed carbon dioxide: a pilot study. Arch Environ Health 1997; 52: 334-340.

33 Shusterman D. Review of the upper airway, including olfaction, as mediator of symptoms. Environ Health Perspect 2002; 110: Suppl. 4, 649-653.

34 Tilles SA. Vocal cord dysfunction in children and adolescents. Curr Allergy Asthma Rep 2003; 3: 467-472.

35 Goldberg BJ, Kaplan MS. Non-asthmatic respiratory symptomatology. Curr Opin Pulm Med 2000; 6: 26-30.

36 Balkissoon R. Disorders of the upper airway. In: Mason RJ et al. eds. Murray and Nadel's Text Book Of Respiratory Medicine. 5th Edn. New York, Elseviers, 2010; pp. 1047-1065.

37 Perkner JJ, Fennelly KP, Balkissoon R, et al. Irritant-associated vocal cord dysfunction. J Occup Environ Med 1998; 40: 136-143.

38 Balkissoon R. Occupational upper airway disease. Clin Chest Med 2002; 23: 717-725.

39 Ayres JG, Gabbott PL. Vocal cord dysfunction and laryngeal hyperresponsiveness: a function of altered autonomic balance? Thorax 2002; 57: 284-285.

40 Kenn K, Freitag L. Endospirometry - development of a device to objectify vocal cord dysfunction and proposal for a classification system. Eur Respir J 2006; 28: Suppl. 50, 793 S.

41 Clerf LH. Unilateral vocal cord paralysis. J Am Med Assoc 1953; 151: 900-903.

42 Parnell FW, Brandenburg JH. Vocal cord paralysis. A review of 100 cases. Laryngoscope 1970; 80: 1036-1045.

43 Nishino T. Physiological and pathophysiological implications of upper airway reflexes in humans. Jpn J Physiol 2000; 50: 3-14.

44 Riley RH, Musk MT. Laryngospasm induced by topical application of lignocaine. Anaesth Intensive Care 2005; 33: 278.

45 Leo RJ, Konakanchi R. Psychogenic respiratory distress: a case of paradoxical vocal cord dysfunction and literature review. Prim Care Companion J Clin Psychiatry 1999; 1: 39-46.

46 Tarlo SM, Poonai N, Binkley K, et al. Responses to panic induction procedures in subjects with multiple chemical sensitivity/idiopathic environmental intolerance: understanding the relationship with panic disorder. Environ Health Perspect 2002; 110: Suppl. 4, 669-671.

47 Poonai NP, Antony MM, Binkley KE, et al. Psychological features of subjects with idiopathic environmental intolerance. J Psychosom Res 2001; 51: 537-541.

48 Poonai N, Antony MM, Binkley KE, et al. Carbon dioxide inhalation challenges in idiopathic environmental intolerance. J Allergy Clin Immunol 2000; 105: 358-363.

49 Bailer J, Witthöft M, Paul C, et al. Evidence for overlap between idiopathic environmental intolerance and somatoform disorders. Psychosom Med 2005; 67: 921-929.

50 Andrianopoulos MV, Gallivan GJ, Gallivan KH. PVCM, PVCD, EPL, and irritable larynx syndrome: what are we talking about and how do we treat it? J Voice 2000; 14: 607-618.

51 Reisner C, Borish L. Heliox therapy for acute vocal cord dysfunction. Chest 1995; 108: 1477.

52 Reybet-Degat O. Pathology of craniocervical junction and sleep disorders. Rev Neurol 2001; 157: 156-160.

53 Maillard I, Schweizer V, Broccard A, et al. Use of botulinum toxin type A to avoid tracheal intubation or tracheostomy in severe paradoxical vocal cord movement. Chest 2000; 118: 874-877. 